\title{
Seismic study of the Herbig Ae star HD 104237
}

\author{
M.-A. Dupret ${ }^{1}$, T. Böhm ${ }^{2}$, M.-J. Goupil ${ }^{1}$, C. Catala ${ }^{1}$ and A. Grigahcène ${ }^{3}$ \\ ${ }^{1}$ Observatoire de Paris, LESIA, CNRS UMR 8109, 92195 Meudon, France \\ ${ }^{2}$ Observatoire Midi-Pyrénées, CNRS, 31400 Toulouse, France \\ ${ }^{3}$ CRAAG - Algiers Observatory BP 63 Bouzareah 16340, Algiers, Algeria
}

\begin{abstract}
HD 104237 is the brightest Herbig Ae star, and as such a pre-main sequence (PMS) star. It is also a spectroscopic binary with determined orbital parameters. It was observed in 1999-2000 at SAAO and Mt Stromlo and 1888 échelle spectra were obtained. From these observations, 8 pulsation frequencies were detected among which 5 with very high confidence (Böhm et al. 2004). We present here a preliminary seismic study based on these observations. We also consider the problem of the excitation of the observed modes and show, as an important result of our study, that HD 104237 is not a $\delta$ Sct type pulsator.
\end{abstract}

\section{Herbig Ae/Be stars}

Herbig Ae/Be stars are PMS objects. Their observational definition (Herbig 1960) tells us that they have spectral types earlier than F0, emission lines are present, they are in an embedded region and close to a reflection nebula. They have intermediate masses $\left(1.5\right.$ to $\left.8 \mathrm{M}_{\odot}\right)$ and present signs of intense activity (jets, outflow, IR excess, X-ray sources, ...) and strong stellar winds.

\section{HD 104237}

HD 104237 (DX Cha) is a bright Herbig Ae $\left(m_{V}=6.6\right)$ and as such it has been observed and studied by many authors. It is the primary of a binary system whose orbital parameters are well determined, the mass ratio is $1.29 \pm 0.02$. Its global parameters are poorly known. van den Ancker et al. (1998) (VA98) give $T_{\text {eff }}=8500 \mathrm{~K}$ and $\log \left(L / L_{\odot}\right)=1.55$, while Grady et al. (2004) (GR04) give $T_{\text {eff }}=7400 \mathrm{~K}$ and $\log \left(L / L_{\odot}\right)=1.41$. Spectroscopic determinations by Böhm et al. (in preparation) will enable us to know these quantities with more precision; very preliminary results favor $T_{\text {eff }}=8250 \mathrm{~K}$. Abundance determinations are going to be performed soon by Böhm et al., no values for $Z$ are available to date. We give in Fig. 1 the position in the HR diagram of HD 104237 corresponding to the determinations of VA98 and GR04. 


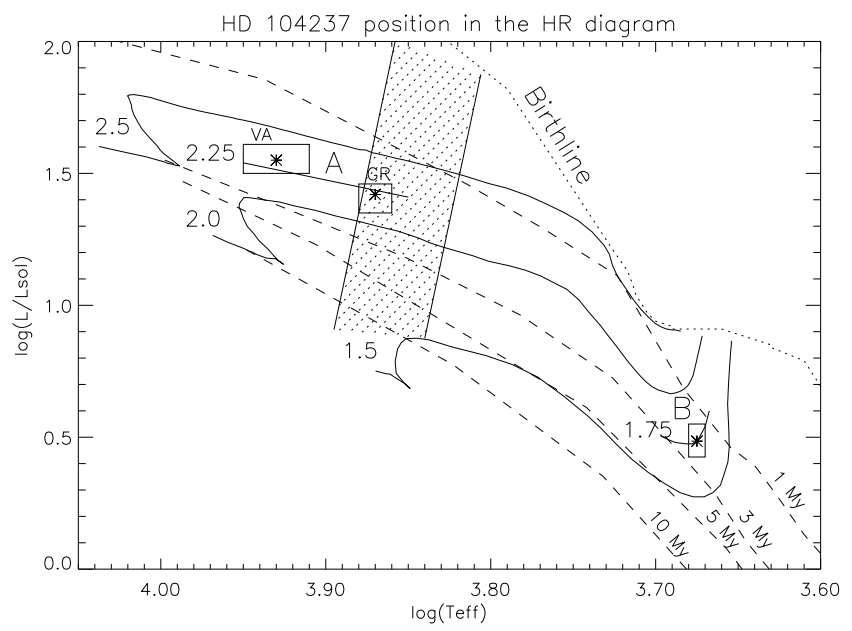

Figure 1: Location of HD 104237 in the HR diagram (VA98 and GR04 determinations).

\section{Seismic observations}

Donati et al. (1997) was the first to detect pulsational variability in HD 104237, and Kurtz \& Müller (1999) confirmed the presence of two close pulsation frequencies. But the revolution opening the way to a real seismic study comes from the observations by Böhm et al. (2004). They observed the star during two distinct runs in 1999 and 2000, over a total of 42 nights at the $1.9 \mathrm{~m}$ Radcliffe telescope of the South African Astronomical Observatory (SAAO) and the Australian 74 inch telescope at Mt. Stromlo. A total of 1888 individual high resolution échelle spectra was gathered. From these observations, 8 pulsation frequencies were detected among which 5 with very high confidence. We present the results of the frequency analysis for the two distinct runs in Table 1. In addition to the 8 pulsation modes, Böhm et al. detected recently in their data set the signature of a signal at $95 \mathrm{hrs}$ that corresponds probably to rotational modulation.

Table 1: Frequencies and amplitudes derived from the Fourier analysis of the 1999 (left) and 2000 (right) data. The uncertainty of the frequency is less than $0.04 \mathrm{c} / \mathrm{d}$ (Böhm et al. 2004).

\begin{tabular}{lcc|ccc}
\hline $\begin{array}{l}\text { Frequency } \\
(\mathrm{c} / \mathrm{d})\end{array}$ & $\begin{array}{c}1999 \\
\text { Amplitude } \\
\left(\mathrm{km} \mathrm{s}^{-1}\right)\end{array}$ & $\begin{array}{c}\text { Conf. } \\
>\%\end{array}$ & $\begin{array}{c}\text { Frequency } \\
(\mathrm{c} / \mathrm{d})\end{array}$ & $\begin{array}{c}2000 \\
\text { Amplitude } \\
\left(\mathrm{km} \mathrm{s}^{-1}\right)\end{array}$ & $\begin{array}{c}\text { Conf. } \\
>\%\end{array}$ \\
\hline $\mathrm{f}_{1}: 33.289$ & 1.32 & 99.9 & 33.283 & 0.26 & 99.9 \\
$\mathrm{f}_{2}: 35.606$ & 0.47 & 99.9 & 35.609 & 0.33 & 99.9 \\
$\mathrm{f}_{3}: 28.503$ & 0.20 & 99.9 & 28.521 & 0.17 & 99.9 \\
$\mathrm{f}_{4}: 30.954$ & 0.14 & 99.0 & 31.012 & 0.18 & 99.9 \\
$\mathrm{f}_{5}: 33.862$ & 0.10 & 99.0 & & & \\
$\mathrm{f}_{6}: 32.616$ & 0.11 & 99.0 & 32.375 & 0.11 & 99.9 \\
$\mathrm{f}_{7}: 34.88$ & 0.10 & & & & \\
$\mathrm{f}_{8}: 35.28$ & 0.05 & & & & \\
\hline
\end{tabular}




\section{Seismic modeling}

We present here a preliminary seismic study of HD 104237 based on the observations of Böhm et al. (2004). The numerical tools used for this study are the following. For the oscillation computations, we use the non-adiabatic code MAD developed by Dupret M.-A. and including the time-dependent convection treatment of Grigahcène et al. (2005). The stellar models were computed with the code CESAM (Morel 1997).

\subsection{Frequency fitting}

Two preliminary things are ideally required for a complete seismic study: a good knowledge of the global parameters of the star and a good idea of $\ell$ and $m$ for each observed mode; however it is seldom the case. In HD 104237, the effective temperature and the luminosity are poorly known and we have no mode identification. On the positive side, we have an estimation of the rotation period ( $95 \mathrm{hrs}$ ), which enables us to determine the frequency splittings due to rotation. In all the models considered in this study, we assume $Z / X=0.0245$ and the solar calibrated values $X=0.7143$ and $\alpha=1.752$.
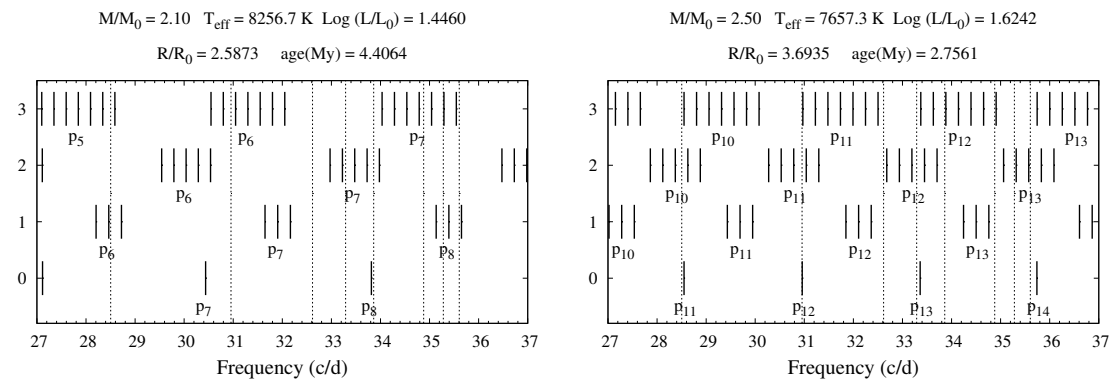

Figure 2: Theoretical and observed frequencies for two models of HD 104237.

A first result we find is that, for models with $T_{\text {eff }}$ and $L$ corresponding to the values proposed by VA98 and GR04, it is difficult to find a good agreement between the theoretical and observed frequencies. We give as an example in the left panel of Fig. 1 the theoretical and observed frequency spectra obtained for a model with $T_{\text {eff }}=8250 \mathrm{~K}$. We see that the theoretical frequency spectrum is sparse for this model and we would have to consider modes with $\ell$ up to 4,5 to be able to fit the observations.

A denser theoretical frequency spectrum would be required for a better fit. Moreover, we remark an interesting feature in the observed frequency spectrum: there is an approximate equidistance around $2.3 \mathrm{c} / \mathrm{d}$. More precisely, we have for the 1999 frequencies $f_{2}-f_{1}=$ $2.451 \mathrm{c} / \mathrm{d}, f_{4}-f_{2}=2.335 \mathrm{c} / \mathrm{d}, f_{7}-f_{4}=2.317 \mathrm{c} / \mathrm{d}, f_{6}-f_{3}=2.264 \mathrm{c} / \mathrm{d}$. This could be the large separation $\Delta f$, but the theoretical values are too large for models with VA98's or GR04's $T_{\text {eff }}$ and $L$. As $\Delta f$ is proportional to $\sqrt{M / R^{3}}$, we have thus to increase the stellar radius for a better fit. However, the radius of PMS stars does not change much during their crossing of the HR diagram. For a sequence with $M=2.3 \mathrm{M}_{\odot}$, we have to decrease $T_{\text {eff }}$ down to $6320 \mathrm{~K}$ to fit $\Delta f$, which is not acceptable. Another possibility is to increase the radius by increasing the mass. To have the good radius, we have to go up to $M=2.5 \mathrm{M}_{\odot}$, in the range of $T_{\text {eff }} \in[7200 \mathrm{~K}, 7700 \mathrm{~K}]$. An example of the good fit obtained in this case is given in the right panel of Fig. 1 . We see that the luminosity of this model is higher than 
the determinations of VA98 and GR04. Its $T_{\text {eff }}$ agrees with the GR04's determination but it is smaller than the VA98's ones.

\subsection{Excitation of modes}

Our non-adiabatic pulsation code enables us to study the mode excitation. However, it appears that in HD 104237 the excitation cannot be explained by standard mechanisms.

Some models of HD 104237 fall inside the classical instability strip ( $\kappa$-driving in the $\mathrm{He}_{\mathrm{II}}$ partial ionization zone), but the modes excited by this mechanism have too small frequencies (typically 5 to $18 \mathrm{c} / \mathrm{d}, \mathrm{p}_{1}-\mathrm{p}_{6}$ modes). This is incompatible with the observed frequencies which correspond to $p_{11}-p_{15}$ modes inside the instability strip. We conclude that HD 104237 is not a standard $\delta$ Sct-type pulsator. For the observed modes of HD 104237, the main driving and damping occurs between the $\mathrm{He}_{\mathrm{II}}$ and $\mathrm{He}_{\mathrm{I}}$ partial ionization zones. We studied if the mode excitation can be explained by turbulent pressure variations in the overshooting region predicted there by non-local convection models (see Dupret et al., these proceedings), but the answer is negative.

On the contrary, if we admit that $T_{\text {eff }} \simeq 8250 \mathrm{~K}$, then the observed modes have smaller radial orders $\left(\mathrm{p}_{6}-\mathrm{p}_{8}\right)$ and could maybe be excited by a mechanism similar to the case of roAp stars (see Theado et al., these proceedings). Further work is required to test if such mechanism could really apply to Herbig Ae stars. In particular, the question is to determine if diffusion has already the time to enhance the $\mathrm{He}$ and $\mathrm{H} \kappa$-driving of the high-order p-modes in so young objects.

\section{Conclusions}

The numerous pulsation frequencies detected in HD 104237 give a unique opportunity to probe the internal structure of a young pre-main sequence star. More work is required to understand the driving of the modes, which cannot be the classical $\kappa$-mechanism. HD 104237 is thus not a standard $\delta$ Sct type pulsator. The equidistance found in the frequency spectrum could be perhaps the large separation. Luminosities higher than the ones determined by VA98 are required to fit this large separation. Better spectroscopic determinations of $T_{\text {eff }}$ and $\log g$ will be performed soon by Böhm et al., which will allows us to go further in our seismic study and better constrain the internal physics of this star.

Acknowledgments. MAD acknowledges financial support by CNES.

\section{References}

Böhm, T., Catala, C., Balona, L., Carter, B. 2004, A\&A 427, 907

Donati, J.F., Semel, M., Carter, B.D., Rees, D.E., Collier Cameron, A. 1997, MNRAS 291, 658

Grady, C.A., Woodgate, B., Torres Carlos, A.O., et al. 2004, ApJ 608, 809

Grigahcène, A., Dupret, M.-A., Gabriel, M., et al. 2005, A\&A 434, 1055

Herbig, G.H. 1960, ApJS 4, 337

Kurtz, D.W., Müller, M. 1999, MNRAS 310, 1071

Morel, P. 1997, A\&AS 124, 597

van den Ancker, M.E., de Winter, D., Tjin A Djie, H.R.E. 1998, A\&A 330, 145 (VA98) 